\title{
Influence of the environment on charge quantization in small superconducting islands
}

\author{
Frank Neumann \\ Fachbereich Physik, Universität-Gesamthochschule Essen, D-45117 Essen, Germany \\ Gert-Ludwig Ingold* \\ Fachbereich Physik, Universität-Gesamthochschule Essen, D-45117 Essen, Germany \\ and Service de Physique de l'Etat Condensé, Commissariat à l'Energie Atomique, Saclay, \\ F-91191 Gif-sur-Yvette Cedex, France \\ Hermann Grabert \\ Fachbereich Physik, Universität-Gesamthochschule Essen, D-45117 Essen, Germany
} and Fakultät für Physik, Universität Freiburg, Hermann-Herder-Strasse 3, D-79104 Freiburg, Germany

(Received 13 April 1994)

\begin{abstract}
The number of Cooper pairs on a small superconducting island coupled to superconducting leads by a Josephson junction fluctuates due to tunneling of Cooper pairs through the junction. On the other hand, the impedance of the circuit surrounding the island tends to suppress these fluctuations and to restore charge quantization. We consider the influence of the electromagnetic environment within a two-state approximation for the charge on the island. The average charge and the slope of the steps of the Coulomb staircase are calculated for a realistic environmental impedance and finite temperatures in the range of current experimental studies. It is shown that even a low-frequency environment may lead to a considerable reduction of charge fluctuations provided the integrated total impedance is large enough.
\end{abstract}

\section{INTRODUCTION}

Recently, clear Coulomb staircase patterns have been observed for metallic islands in the normal state charged one by one with electrons ${ }^{1}$ as well as for superconducting metallic islands charged by Cooper pairs. ${ }^{2}$ Aside from the electrostatic energy responsible for Coulomb blockade phenomena, ${ }^{3}$ a more detailed theory has to include effects due to quantum fluctuations of the electric charge and the electromagnetic environment. ${ }^{4}$ In view of possible applications of single charge phenomena for high precision devices, ${ }^{5}$ these effects are of considerable importance, even if they are not dominant. The simplest system to study the influence of charge fluctuations on the Coulomb staircase is the so-called box where an island is formed by a tunnel junction and a capacitor. If the charging energy of the island is the dominant energy in the system, quantization of the island charge can be expected. However, thermal fluctuations and the coupling of the island to the external leads due to the tunnel junction tend to suppress charge quantization. ${ }^{6}$ Here, we focus on the influence of the electromagnetic environment on the behavior of a superconducting box circuit.

In Sec. II we introduce the Hamiltonian describing a superconducting island coupled via a Josephson junction to its electromagnetic environment. For a study of charge fluctuations at low temperatures, it is often sufficient to restrict oneself to two charge states and perform a mapping to a spin-boson problem as shown in Sec. III. Information about the average island charge is contained in the partition function which is calculated in
Sec. IV for an environment with a low-frequency part of arbitrary strength and a small high-frequency contribution. This corresponds to the form of the environmental impedance in realistic set-ups. In Secs. V and VI we separately consider the influence of the low-frequency and high-frequency parts of the impedance on charge quantization. Finally, in Sec. VII we present our conclusions.

\section{DESCRIPTION OF THE ENVIRONMENT}

We consider the superconducting circuit shown in Fig. 1 where an island is formed by a gate capacitor and a Josephson junction opening the possibility to charge the island by tunneling. The Josephson junction is charac-

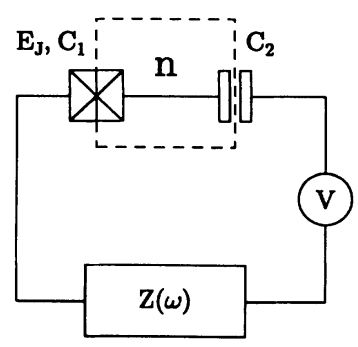

FIG. 1. Superconducting island formed by a small Josephson junction with Josephson coupling energy $E_{J}$ and capacitance $C_{1}$ and a capacitor with capacitance $C_{2}$. The island is coupled to a voltage source via an impedance $Z(\omega)$. The number of excess Cooper pairs on the island is denoted by $n$. 
terized by the Josephson coupling energy $E_{J}$ and the capacitance $C_{1}$ while the gate capacitor has a capacitance $C_{2}$. In the parameter region studied here (see below) quasiparticles may be neglected and the island charge may then be expressed in terms of the number of excess Cooper pairs $n$ on the island as

$$
q_{\text {island }}=-2 n e=Q_{1}-Q_{2}
$$

where $Q_{1}$ and $Q_{2}$ are the charges on the Josephson junction and on the gate capacitor, respectively.

The island charge may be controlled by means of an externally applied voltage $V$. It is convenient to introduce the voltage source as a capacitor of infinite capacitance $C_{v}$ carrying the charge $C_{v} V$. Combining this capacitor with the gate capacitor one gets a capacitor of capacitance $C_{2}$ carrying the charge $Q_{2}-C_{2} V$. The influence of the voltage source on the island charge may then be expressed in terms of an external charge $2 e n_{x}$ with $n_{x}=C_{2} V / 2 e$ so that we get for the effective island charge in the presence of an external voltage source

$$
q=-2 e\left(n-n_{x}\right)
$$

Introducing the island capacitance $C_{\Sigma}=C_{1}+C_{2}$, the Hamiltonian describing the charging energy of the island may be written as

$$
H_{c}=E_{c}\left(n-n_{x}\right)^{2},
$$

where $E_{c}=2 e^{2} / C_{\Sigma}$ is the charging energy of a single Cooper pair. For vanishing Josephson coupling and vanishing temperature and in the absence of an external impedance, minimization of the charging energy following from (3) leads to a Coulomb staircase ${ }^{7}$ for the number of Cooper pairs on the island as a function of the external charge with steps at $n_{x}= \pm 1 / 2, \pm 3 / 2, \ldots$. Both finite Josephson coupling and finite temperatures cause charge fluctuations and a corresponding washout of the steps. To avoid thermal smearing we assume that the charging energy is much larger than the thermal energy, i.e., $E_{c} \gg k_{B} T$.

The expression (3) for the charging energy and therefore also the positions of the steps of the Coulomb staircase are only correct in the absence of quasiparticles. If the gap energy $\Delta$, which is the minimal energy associated with the formation of a quasiparticle, is sufficiently small, i.e., $\Delta<E_{c} / 4$, the system may decrease its energy in certain voltage ranges by having a single quasiparticle on the island. In this paper we restrict ourselves to the case where $\Delta \gg E_{c}$ so that quasiparticles may safely be neglected.

In the following we will account for an external impedance $Z(\omega)$ of the circuit as indicated in Fig. 1. Before writing down a Hamiltonian for this electromagnetic environment it is convenient to employ a circuit transformation to simplify the circuit. It can be shown ${ }^{4}$ that seen from the Josephson junction the circuit presented in Fig. 2 is equivalent to the circuit in Fig. 1. The capacitor with capacitance $C_{\Sigma}$ corresponds to the island as discussed above. The total impedance

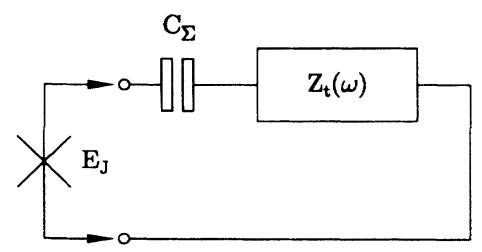

FIG. 2. Circuit of Fig. 1 as seen from the Josephson junction. $C_{\Sigma}=C_{1}+C_{2}$ is the island capacitance and $Z_{t}(\omega)$ is the total impedance as defined in (4).

$$
Z_{t}(\omega)=\kappa^{2} \frac{1}{i \omega C+Z(\omega)^{-1}}
$$

corresponds, apart from a factor $\kappa^{2}<1$ with

$$
\kappa=\frac{C_{2}}{C_{1}+C_{2}}
$$

to the impedance of the total capacitance $C=$ $C_{1} C_{2} /\left(C_{1}+C_{2}\right)$ in parallel with the external impedance $Z(\omega)$. The total impedance may now be modeled by a Caldeira-Leggett Hamiltonian describing a set of harmonic oscillators 8

$$
H_{\mathrm{env}}=\sum_{m=1}^{N}\left[\frac{Q_{m}^{2}}{2 C_{m}}+\frac{1}{2 L_{m}}\left(\frac{\hbar}{2 e} \varphi_{m}\right)^{2}\right],
$$

where the charges $Q_{j}$ and phases $\varphi_{k}$ are conjugate operators with $\left[Q_{j}, \varphi_{k}\right]=-2 i e \delta_{j k}$. In terms of an electric circuit model the environmental Hamiltonian (6) describes a collection of $L C$ circuits with frequencies $\omega_{m}=\left(L_{m} C_{m}\right)^{-1 / 2}$ in series, and

$$
U_{m}=(\hbar / 2 e) \dot{\varphi}_{m}
$$

is the voltage across the $m$ th $L C$ circuit. The corresponding total impedance is given by

$$
Z_{t}(\omega)=\sum_{m=1}^{N} \frac{1}{C_{m}} \frac{i \omega}{\omega_{m}^{2}-\omega^{2}}
$$

The Hamiltonian (6) can represent any given total impedance if the set of parameters $C_{m}$ and $L_{m}$ is chosen appropriately.

The tunneling of Cooper pairs to and from the island is described by the Josephson term $-E_{J} \cos (\varphi)$ where $\varphi$ is the phase difference across the junction. By means of the Josephson relation

$$
\dot{\varphi}=\frac{2 e}{\hbar} U
$$

the time derivative of the phase $\varphi$ is connected with the voltage $U$ across the junction. In addition, we introduce the phase $\psi$ which is conjugate to the number of Cooper pairs on the island, i.e., $[\psi, n]=-i$. Therefore,

$$
n e^{-i \psi}=e^{-i \psi}(n+1) \text {, }
$$

so that the operator $e^{-i \psi}$ describes the tunneling of a Cooper pair onto the island. $\psi$ is chosen in such a way that it fulfills (9) with $U$ replaced by $-2 e\left(n-n_{x}\right) / C_{\Sigma}$. 
Since the voltages in the circuit loop of Fig. 2 have to add up to zero, we may express the phase $\varphi$ across the Josephson junction in terms of the phase $\psi$ across the capacitor and the phases $\varphi_{m}$ associated with the $L C$ circuits of the total impedance as

$$
\varphi=-\psi-\sum_{m=1}^{N} \varphi_{m}
$$

The tunneling part of the Hamiltonian then becomes

$$
\begin{aligned}
H_{T}= & -\frac{E_{J}}{2}\left[\exp \left(i \psi+i \sum_{m=1}^{N} \varphi_{m}\right)\right. \\
& \left.+\exp \left(-i \psi-i \sum_{m=1}^{N} \varphi_{m}\right)\right] .
\end{aligned}
$$

Here, the terms with $\psi$ change the island charge as shown above while the phases $\varphi_{m}$ give rise to a coupling of the tunneling process to the environment. The Hamiltonian describing the circuit in Fig. 1 is then given by (3), (6), and (12) as

$$
H=H_{c}+H_{\mathrm{env}}+H_{T} .
$$

As already mentioned, the number $n$ of excess Cooper pairs on the island at a fixed voltage bias is in general not constant but will fluctuate. The average number of Cooper pairs may be calculated from

$$
\langle n\rangle=n_{x}+\frac{1}{2 \beta E_{c}} \frac{\partial}{\partial n_{x}} \ln (\mathcal{Z}),
$$

where we have introduced the reduced partition function

$$
\mathcal{Z}=\frac{\operatorname{Tr} e^{-\beta H}}{\operatorname{Tr} e^{-\beta H_{\text {env }}}} .
$$

\section{MAPPING ONTO THE SPIN-BOSON-PROBLEM}

Only in the limit of vanishing Josephson coupling and zero temperature is the number $n$ of excess Cooper pairs on the island a strict staircase function of $n_{x}$. In general, the island charge will fluctuate and we have to consider the average number of Cooper pairs $\langle n\rangle$ as a function of $n_{x}$. The fluctuations of $n$ will lead to a broadening of the steps. Since we are mainly interested in the influence of the environment on the fluctuations of the island charge, we focus on the vicinity of a step and study the dependence of $\langle n\rangle$ on the external impedance for values of $n_{x}$ close to this step. The Hamiltonian (13) is invariant under the transformation $n_{x} \rightarrow n_{x}+1$ and $n \rightarrow n+1$. Therefore, all steps have the same shape but neighboring steps are shifted in $\langle n\rangle$ by 1 . In the following we will choose the step at $n_{x}=1 / 2$ and consider especially the slope

$$
\chi=\left.\frac{\partial\langle n\rangle}{\partial n_{x}}\right|_{n_{x}=1 / 2} .
$$

This quantity will only be large, corresponding to an almost strict charge quantization, if the charge fluctuations are small.
In the vicinity of $n_{x}=1 / 2$ the most relevant island charge states will be $n=0$ and $n=1$. Restricting ourselves to these two states, the island charge operator $n$ may be replaced by

$$
n=\frac{1-\sigma_{z}}{2},
$$

with the Pauli matrix

$$
\sigma_{z}=\left(\begin{array}{rr}
1 & 0 \\
0 & -1
\end{array}\right)
$$

The Coulomb charging term $H_{c}$ in the Hamiltonian (13) may then be written as

$$
H_{c}=E_{c} \epsilon \sigma_{z},
$$

where

$$
\epsilon=n_{x}-\frac{1}{2}
$$

gives the distance from the step at $n_{x}=1 / 2$. In the following $\epsilon$ will be regarded as small compared to 1 . In (19) we have omitted an energy term $E_{c}\left(1 / 4+\epsilon^{2}\right)$ which depends on $\epsilon$. Therefore, in the following we have to use

$$
\langle n\rangle=\frac{1}{2}+\frac{1}{2 \beta E_{c}} \frac{\partial}{\partial n_{x}} \ln (\mathcal{Z})
$$

instead of (14).

In view of (10) the part (12) of the Hamiltonian describing the tunneling of Cooper pairs and its coupling to the environment becomes

$$
H_{T}=-\frac{E_{J}}{2}\left(\begin{array}{cc}
0 & \exp \left(i \sum_{m=1}^{N} \varphi_{m}\right) \\
\exp \left(-i \sum_{m=1}^{N} \varphi_{m}\right) & 0
\end{array}\right)
$$

The environmental part (6) of the Hamiltonian remains unchanged. The total Hamiltonian is now given by (13) with the definitions (19), (6), and (22)..$^{9}$ By means of the unitary transformation

$$
U=\left(\begin{array}{cc}
0 & \exp \left(-i \sum_{m=1}^{N} \varphi_{m} / 2\right) \\
\exp \left(i \sum_{m=1}^{N} \varphi_{m} / 2\right) & 0
\end{array}\right)
$$

it can be shown that the new Hamiltonian is equivalent to the spin-boson Hamiltonian in its usual form ${ }^{10}$ with a spectral density of bath oscillators

$$
J(\omega)=\lim _{\varepsilon \rightarrow 0} \omega \operatorname{Re}\left[Z_{t}(\omega-i \varepsilon)\right]
$$

It is now straightforward to calculate the partition function (15) which may be written as

$$
\mathcal{Z}=\mathcal{Z}_{0}(\epsilon)+\mathcal{Z}_{1}(\epsilon)
$$

where the two contributions $\mathcal{Z}_{0}$ and $\mathcal{Z}_{1}$ correspond to the uncharged island and the island with one additional 
Cooper pair, respectively. From the definition (20) of $\epsilon$ it is clear that $\mathcal{Z}_{1}(\epsilon)=\mathcal{Z}_{0}(-\epsilon)$. Expanding the partition function in powers of the Josephson coupling energy one finds

$$
\mathcal{Z}_{0}=\sum_{m=0}^{\infty}\left(\frac{E_{J}}{2 \hbar}\right)^{2 m} \int_{0}^{\hbar \beta} d s_{2 m} \int_{0}^{s_{2 m}} d s_{2 m-1} \cdots \int_{0}^{s_{2}} d s_{1} \exp \left[-z_{m}\left(s_{1}, \ldots, s_{2 m}\right)\right]
$$

with

$$
z_{m}\left(s_{1}, \ldots, s_{2 m}\right)=\left(\hbar \beta-2 s_{2 m}+2 s_{2 m-1}-\cdots-2 s_{2}+2 s_{1}\right) \frac{E_{c}}{\hbar} \epsilon+\sum_{k=2}^{2 m} \sum_{\ell=1}^{k-1}(-1)^{k+\ell} \Lambda\left(s_{k}-s_{\ell}\right)
$$

Here, we have introduced the function

$$
\begin{aligned}
\Lambda(t)= & 2 \int_{0}^{\infty} \frac{d \omega}{\omega} \frac{\operatorname{Re} Z_{t}(\omega)}{R_{Q}}\left[\operatorname{coth}\left(\frac{\beta \hbar \omega}{2}\right)[\cosh (\omega t)-1]\right. \\
& -\sinh (\omega t)]
\end{aligned}
$$

which obeys the symmetry

$$
\Lambda(\hbar \beta-t)=\Lambda(t)
$$

Note that the relevant impedance scale for $Z_{t}(\omega)$ is set by the resistance quantum $R_{Q}=h / 4 e^{2} \sim 6.45 \mathrm{k} \Omega$. The function $\Lambda(t)$ is the imaginary time continuation of the phase correlation function which determines the influence of the environment on transport properties of ultrasmall tunnel junctions. ${ }^{11,4}$

Before proceeding with the calculation we have to discuss the conditions under which the restriction to two charge states is allowed. Of course, the two lowest states should energetically be well separated from the higher states. This can only be the case if one is close to a step, i.e., $\epsilon \ll 1$. Then the energy difference between the two lowest states is given by the Josephson coupling energy $E_{J}$ while the distance to the next higher level is determined by the charging energy $E_{c}$. Therefore, our approximation requires $E_{J} \ll E_{c}$. Furthermore, excitations to higher levels should be negligible. Hence, the temperature should be low enough so that $k_{B} T \ll E_{c}$. Also, high-frequency environmental modes may invalidate the two-state approximation. However, modes with frequencies much larger than $E_{c} / \hbar$ are suppressed by the capacitive cutoff in the total impedance (4) due to the total capacitance $C$.

\section{PARTITION FUNCTION}

The impedance at frequencies of the order of $E_{c} / \hbar$, which lies in the $\mathrm{GHz}$ range or above, is usually small compared to the resistance quantum. On the other hand, at low frequencies the impedance may become quite large. ${ }^{12}$ We therefore decompose the total impedance $Z_{t}(\omega)$ into a low-frequency contribution $Z_{1}(\omega)$ of arbitrary strength and a small high-frequency impedance $Z_{2}(\omega)$ as shown in Fig. 3. Accordingly, we write the imaginary time correlation function $\Lambda(t)$ introduced in (28) as $\Lambda(t)=\Lambda_{1}(t)+\Lambda_{2}(t)$.
Before evaluating the partition function given by (25) and (26), we discuss the low-frequency part $Z_{1}(\omega)$ of the total impedance. While $\operatorname{Re} Z_{t}(\omega)$ may well exceed $R_{Q}$ at low frequencies, the impedance typically falls off in the $\mathrm{kHz}-\mathrm{MHz}$ range. Even for temperatures in the $\mathrm{mK}$ range this cutoff frequency is small compared to $k_{B} T / \hbar$. Since $t$ is always smaller than $\hbar \beta$, we may replace $\Lambda_{1}(t)$ by its short-time expansion which is obtained from (28) as

$$
\Lambda_{1}(t)=\frac{2 a}{\hbar \beta} t(t-\hbar \beta)
$$

where

$$
a=\int_{0}^{\infty} d \omega \frac{\operatorname{Re} Z_{1}(\omega)}{R_{Q}}
$$

is the integrated impedance. Inserting (30) into (27) one finds

$$
z_{m}\left(s_{1}, \ldots, s_{2 m}\right)=(\hbar \beta-2 t) \frac{E_{c}}{\hbar} \epsilon+\frac{2 a}{\hbar \beta} t(\hbar \beta-t),
$$

which only depends on the combination

$$
t=\sum_{k=1}^{m}\left(s_{2 k}-s_{2 k-1}\right)
$$

of the times $s_{i}$.

On the other hand, the high-frequency impedance $Z_{2}(\omega)$ is assumed to be small compared to the resistance quantum. Hence, we may expand the partition function (26) to first order in $\Lambda_{2}(t)$ yielding

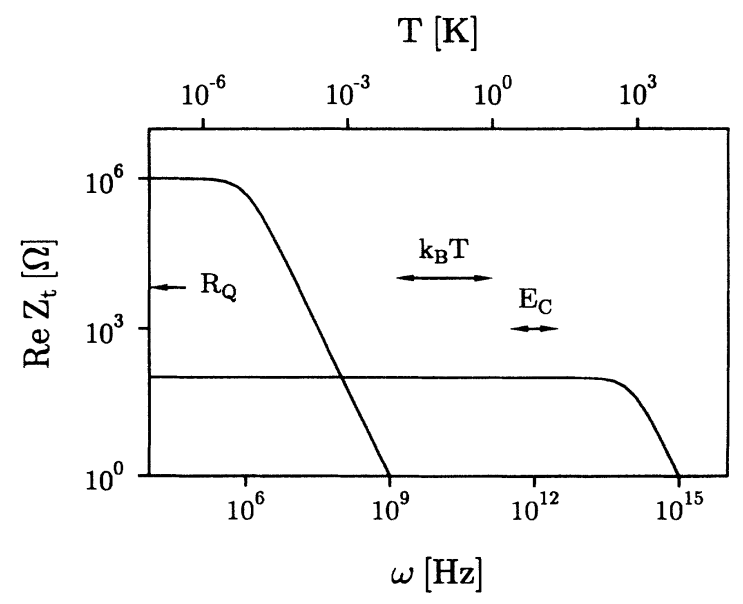

FIG. 3. Frequency dependence of a typical total impedance consisting of a low-impedance part of arbitrary strength and a small contribution at higher frequencies. Typical experimental values for the temperature and the charging energy of the island are indicated. 


$$
\mathcal{Z}_{0}=\sum_{m=0}^{\infty}\left(\frac{E_{J}}{2 \hbar}\right)^{2 m} \int_{0}^{\hbar \beta} d t \exp \left[-(\hbar \beta-2 t) \frac{E_{c}}{\hbar} \epsilon-\frac{2 a}{\hbar \beta} t(\hbar \beta-t)\right] f_{m}(t)
$$

with $f_{0}(t)=\delta(t)$ and

$$
f_{m}(t)=\int_{0}^{\hbar \beta} d s_{2 m} \cdots \int_{0}^{s_{2}} d s_{1} \delta\left(t-\sum_{k=1}^{m}\left(s_{2 k}-s_{2 k-1}\right)\right)\left[1-\sum_{k=2}^{2 m} \sum_{\ell=1}^{k-1}(-1)^{k+\ell} \Lambda_{2}\left(s_{k}-s_{\ell}\right)\right] .
$$

Evaluating the $2 m$ integrals we find

$$
f_{1}(t)=t\left[1+\Lambda_{2}(t)\right]
$$

and for $m \geq 2$

$$
\begin{aligned}
f_{m}(t)= & \frac{1}{m !(m-1) !}(\hbar \beta-t)^{m} t^{m-1} \\
& +\int_{0}^{t} d u \Lambda_{2}(u) \frac{1}{(m-1) !(m-2) !}(\hbar \beta-t)(\hbar \beta-2 u)(t-u)^{m-2}(\hbar \beta-t-u)^{m-2}
\end{aligned}
$$

where we made use of the symmetry (29). We now insert (36) and (37) into (34). Since $\mathcal{Z}_{1}$ is obtained from $\mathcal{Z}_{0}$ by changing the sign of $\epsilon$, we immediately obtain the partition function $\mathcal{Z}$ from (25). Making use of the series expansion of the Bessel function $I_{1}(x)$ we find for the partition function

$$
\begin{aligned}
\mathcal{Z}= & 2 \cosh \left(\beta E_{c} \epsilon\right)+\beta E_{J} \int_{0}^{1} d u\left(1-u^{2}\right)^{-1 / 2} I_{1}\left(\frac{\beta E_{J}}{2}\left(1-u^{2}\right)^{1 / 2}\right) \cosh \left(\beta E_{c} \epsilon u\right) \exp \left(-\frac{\hbar \beta a}{2}\left(1-u^{2}\right)\right) \\
& +\left(\frac{\beta E_{J}}{2}\right)^{2} \int_{0}^{1} d u \Lambda_{2}\left(\frac{\hbar \beta}{2}(1-u)\right) \cosh \left(\beta E_{c} \epsilon u\right) \exp \left(-\frac{\hbar \beta a}{2}\left(1-u^{2}\right)\right) \\
& +\left(\frac{\beta E_{J}}{2}\right)^{3} \int_{0}^{1} d u u \Lambda_{2}\left(\frac{\hbar \beta}{2}(1-u)\right) \\
& \quad \times \int_{0}^{u} d v \cosh \left(\beta E_{c} \epsilon v\right) \exp \left(-\frac{\hbar \beta a}{2}\left(1-v^{2}\right)\right)\left(u^{2}-v^{2}\right)^{-1 / 2} I_{1}\left(\frac{\beta E_{J}}{2}\left(u^{2}-v^{2}\right)^{1 / 2}\right) .
\end{aligned}
$$

In this result the low-frequency part of the impedance is treated nonperturbatively while the high-frequency part is taken into account to leading order. Note that all orders of the expansion in powers of the Josephson coupling energy $E_{J}$ have been resummed in deriving (38). The formula can easily be evaluated numerically for arbitrary environmental impedances with an overall shape of the form shown in Fig. 3. Simpler expressions can be given in special cases considered in the following two sections.

\section{INFLUENCE OF A LOW-IMPEDANCE ENVIRONMENT}

In this section we will assume that for all frequencies the real part of the total impedance is small compared to the resistance quantum. In this case we may set $a=0$ in (38) and use the perturbative result for the partition function. Introducing

$$
\omega_{0}=\left[\left(\frac{E_{J}}{2 \hbar}\right)^{2}+\left(\frac{E_{c}}{\hbar} \epsilon\right)^{2}\right]^{1 / 2}
$$

and employing the relation

$$
\cosh \left(\beta E_{c} \epsilon u\right)+\frac{\beta E_{J}}{2} u \int_{0}^{u} d v \cosh \left(\beta E_{c} \epsilon v\right)\left(u^{2}-v^{2}\right)^{-1 / 2} I_{1}\left(\frac{\beta E_{J}}{2}\left(u^{2}-v^{2}\right)^{1 / 2}\right)=\cosh \left(\hbar \beta \omega_{0} u\right)
$$

the partition function may be written as

$$
\mathcal{Z}=2 \cosh \left(\hbar \beta \omega_{0}\right)+2\left(\frac{E_{J}}{2 \hbar}\right)^{2} \int_{0}^{\infty} d \omega \frac{\operatorname{Re} Z_{t}(\omega)}{R_{Q}} \frac{2 \hbar \beta}{\omega^{2}-4 \omega_{0}^{2}}\left[\cosh \left(\hbar \beta \omega_{0}\right)-\frac{\omega}{2 \omega_{0}} \sinh \left(\hbar \beta \omega_{0}\right) \operatorname{coth}(\hbar \beta \omega / 2)\right]
$$


in agreement with earlier results for the spin-boson problem. ${ }^{13}$

The first term in (41) corresponds to the partition function in the absence of an environment, i.e., for zero external impedance. In this case we obtain from (21) for the average number of excess Cooper pairs on the island

$$
\langle n\rangle=\frac{1}{2}+\frac{\epsilon E_{c}}{\left(E_{J}^{2}+4 E_{c}^{2} \epsilon^{2}\right)^{1 / 2}} \tanh \left[\beta\left(\frac{E_{J}^{2}}{4}+E_{c}^{2} \epsilon^{2}\right)^{1 / 2}\right],
$$

and the slope of the step (16) is found to read

$$
\chi_{0}=\frac{E_{c}}{E_{J}} \tanh \left(\beta E_{J} / 2\right),
$$

which increases with decreasing Josephson coupling as expected. Note that $\chi_{0}$ goes to zero in the limit of infinite temperature which is an artifact of the two-state approximation. This will also be the case in subsequent results for the slope.

We now allow for a low-impedance environment of the form $\operatorname{Re} Z_{t}(\omega)=Z(0) f\left(\omega / \omega_{R}\right)$ where $\omega_{R}$ is a cutoff frequency. Here, $Z(0) / R_{Q}$ should be finite but small and the cutoff function $f\left(\omega / \omega_{R}\right)$ should take the value $f(0)=1$ at zero frequency and vanish for large frequencies. To first order in the damping strength $Z(0) / R_{Q}$ the effect of a small impedance may be described as a shift of the frequency $\omega_{0}$ according to

$$
\tilde{\omega}=\omega_{0}+\frac{Z(0)}{R_{Q}} \omega_{1}
$$

with

$$
\omega_{1}=\left(\frac{E_{J}}{2 \hbar}\right)^{2} \int_{0}^{\infty} d \omega f\left(\omega / \omega_{R}\right) \frac{2}{\omega^{2}-4 \omega_{0}^{2}}\left[\operatorname{coth}\left(\hbar \beta \omega_{0}\right)-\frac{\omega}{2 \omega_{0}} \operatorname{coth}(\hbar \beta \omega / 2)\right] .
$$

For $\hbar \beta \omega_{R} \gg 1$ we find

$$
\omega_{1}=\frac{1}{\omega_{0}}\left(\frac{E_{J}}{2 \hbar}\right)^{2}\left[\operatorname{Re} \psi\left(i \frac{\hbar \beta \omega_{0}}{2 \pi}\right)-\ln \left(\frac{\hbar \beta \omega_{R}}{2 \pi}\right)-\delta\right]
$$

where

$$
\delta=\int_{0}^{\infty} \frac{d \omega}{\omega}\left[f\left(\omega / \omega_{R}\right)-\frac{1}{1+\left(\omega / \omega_{R}\right)^{2}}\right]
$$

depends on the form of the cutoff function. In the low-impedance limit we thus get from $\mathcal{Z}=2 \cosh (\hbar \beta \tilde{\omega})$ and $(21)$ for the average number of excess Cooper pairs

$$
\langle n\rangle=\frac{1}{2}+\frac{\epsilon E_{c}}{2 \hbar \omega_{0}} \tanh (\hbar \beta \tilde{\omega})\left[2-\frac{\tilde{\omega}}{\omega_{0}}-\frac{Z(0)}{R_{Q}} \frac{\beta E_{J}^{2}}{8 \pi \hbar \omega_{0}} \operatorname{Im} \psi^{\prime}\left(i \frac{\hbar \beta \omega_{0}}{2 \pi}\right)\right] .
$$

The slope at $\epsilon=0$ defined in (16) becomes

$$
\begin{gathered}
\chi=\chi_{0}+\frac{Z(0)}{R_{Q}} \frac{E_{c}}{E_{J}}\left\{\left[\tanh \left(\frac{\beta E_{J}}{2}\right)-\frac{\beta E_{J}}{2} \cosh ^{-2}\left(\frac{\beta E_{J}}{2}\right)\right]\left[\ln \left(\frac{\hbar \beta \omega_{R}}{2 \pi}\right)+\delta-\operatorname{Re} \psi\left(i \frac{\beta E_{J}}{4 \pi}\right)\right]\right. \\
\left.-\frac{\beta E_{J}}{4 \pi} \tanh \left(\frac{\beta E_{J}}{2}\right) \operatorname{Im} \psi^{\prime}\left(i \frac{\beta E_{J}}{4 \pi}\right)\right\},
\end{gathered}
$$

with $\chi_{0}$ given by (43). Hence, for a not too small cutoff frequency $\omega_{R} \gg E_{J} / \hbar$, even a low-impedance environment leads to a reduction of the charge fluctuations on the island.

\section{INFLUENCE OF A LOW-FREQUENCY ENVIRONMENT}

We now concentrate on the effect of a low-frequency impedance which may be of arbitrary strength. The environment is characterized by the quantity $a$ defined in (31). By setting $\Lambda_{2}(t)=0$ we get from (38) for the partition function

$$
\mathcal{Z}=2 \cosh \left(\beta E_{c} \epsilon\right)+\beta E_{J} \int_{0}^{1} d u\left(1-u^{2}\right)^{-1 / 2} I_{1}\left(\frac{\beta E_{J}}{2} u\right) \cosh \left[\beta E_{c} \epsilon\left(1-u^{2}\right)^{1 / 2}\right] \exp \left[-\frac{\hbar \beta a}{2} u^{2}\right]
$$

Before deriving in the remainder of this section some analytical result in various limits, we discuss the general behavior of the average number $\langle n\rangle$ of Cooper pairs on the island. Figure 4 shows the dependence of $\langle n\rangle$ on the voltage $(2 \epsilon+1) e / C_{2}$ close to the step at $\epsilon=0$. The step function corresponds to perfect charge quantization in the limit of vanishing temperature and vanishing Josephson coupling energy. For infinite temperature all charging effects are washed out and we find a straight line. In between, two curves for finite temperature $\beta E_{c}=100$ and 


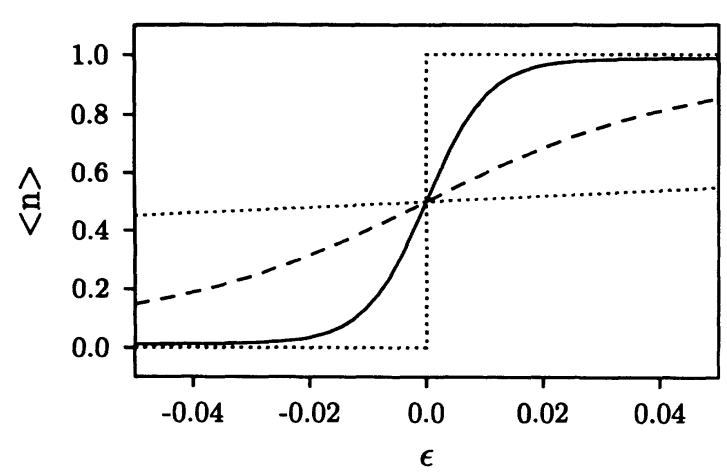

FIG. 4. Voltage dependence of the average number of excess Cooper pairs on the island for voltages close to the step. The two dotted lines represent the limiting cases of vanishing temperature and Josephson coupling energy (step function) and of infinite temperature (straight line). The two other curves correspond to the case of finite temperature $\beta E_{c}=100$ and finite Josephson coupling $E_{J} / E_{c}=0.1$. The dashed line represents the undamped case (zero external impedance) according to (42) while the solid line was calculated for $\hbar a / E_{J}=2$ from (50) using (21).

finite Josephson coupling $E_{J} / E_{c}=0.1$ are shown. In the absence of an external impedance the step is smeared considerably (dashed line). In contrast, the curve calculated numerically from (50) together with (21) and (16) for a low-frequency environment with $\hbar a / E_{J}=2$ (solid line) exhibits a much clearer charge quantization.
We now discuss the partition function (50) and the slope of the step following from it for different regimes of temperature, Josephson coupling energy, and damping strength. We first consider the limit of very strong damping where $\hbar a \gg k_{B} T, E_{J}$. From (50) we then find for the partition function

$$
\mathcal{Z}=\left(2+\frac{\beta E_{J}^{2}}{4 \hbar a}\right) \cosh \left(\beta E_{c} \epsilon\right)
$$

which combines with (21) to yield up to corrections of order $1 / a^{2}$ for the average number of excess Cooper pairs

$$
\langle n\rangle=\frac{1}{2}+\frac{1}{2} \tanh \left(\beta E_{c} \epsilon\right)
$$

and for the slope of the step

$$
\chi=\frac{\beta E_{c}}{2} .
$$

Comparing these results with (42) and (43) we find that a very large integrated impedance (31) entirely suppresses charge fluctuations due to the Josephson coupling.

For sufficiently small Josephson coupling energy with $E_{J} \ll k_{B} T$ we may evaluate the integral in (50) up to order $\left(\beta E_{J}\right)^{2}$. The partition function then becomes

$$
\begin{aligned}
\mathcal{Z}=2 \cosh \left(\beta E_{c} \epsilon\right)+ & \frac{1}{4}\left(\frac{2 \pi}{\hbar \beta a}\right)^{1 / 2}\left(\frac{\beta E_{J}}{2}\right)^{2} \exp \left\{-\frac{\hbar \beta a}{2}\left[1+\left(\frac{E_{c} \epsilon}{\hbar a}\right)^{2}\right]\right\} \\
& \times\left\{\operatorname{erfi}\left[\left(\frac{\hbar \beta a}{2}\right)^{1 / 2}\left(1+\frac{E_{c} \epsilon}{\hbar a}\right)\right]+\operatorname{erfi}\left[\left(\frac{\hbar \beta a}{2}\right)^{1 / 2}\left(1-\frac{E_{c} \epsilon}{\hbar a}\right)\right]\right\}
\end{aligned}
$$

with

$$
\operatorname{erfi}(x)=-i \operatorname{erf}(i x)=\frac{2}{\pi^{1 / 2}} \int_{0}^{x} d t e^{t^{2}}
$$

The derivative of the partition function with respect to $\epsilon$ becomes

$$
\begin{aligned}
\frac{\partial \mathcal{Z}}{\partial \epsilon}= & 2 \beta E_{c} \sinh \left(\beta E_{c} \epsilon\right)\left[1+\frac{1}{2 \hbar \beta a}\left(\frac{\beta E_{J}}{2}\right)^{2}\right] \\
& -\epsilon \frac{\beta E_{c}^{2}}{\hbar a}\left[\mathcal{Z}-2 \cosh \left(\beta E_{c} \epsilon\right)\right],
\end{aligned}
$$

which combines with (21) and (16) to yield for the slope of the step

$$
\chi=\frac{\beta E_{c}}{2}\left[1+\left(\beta E_{J}\right)^{2} g(\hbar \beta a)\right],
$$

where

$$
\begin{aligned}
g(x)= & \frac{1}{16}\left\{\frac{2}{x}-\left(1+\frac{1}{x}\right)\left(\frac{2 \pi}{x}\right)^{1 / 2}\right. \\
& \left.\times \exp \left[-\frac{x}{2}\right] \operatorname{erfi}\left[\left(\frac{x}{2}\right)^{1 / 2}\right]\right\}
\end{aligned}
$$

This function has the expansions

$$
g(x)=-\frac{1}{12}\left(1-\frac{2}{5} x+O\left(x^{2}\right)\right)
$$

and

$$
g(x)=-\frac{1}{4 x^{2}}+O\left(x^{-3}\right) .
$$

Accordingly, for small damping, $\hbar \beta a \ll 1$, the slope becomes

$$
\chi=\frac{\beta E_{c}}{2}-\frac{1}{6} \beta E_{c}\left(\frac{\beta E_{J}}{2}\right)^{2}\left[1-\frac{2}{5} \hbar \beta a\right],
$$


while for strong damping, $\hbar \beta a \gg 1$, one obtains

$$
\chi=\frac{\beta E_{c}}{2}\left[1-\left(\frac{E_{J}}{2 \hbar a}\right)^{2}\right] \text {. }
$$

Finally, we consider the limit of very low and even zero temperature. According to the discussion in Sec. IV the calculation given above is strictly speaking only valid for zero temperature if the impedance has a $\delta$-function-type pole at $\omega=0$. The zero-temperature result should nevertheless give an indication of the behavior of the system at very low temperatures. According to (43), in the absence of an electromagnetic environment the slope of the step in the limit of vanishing temperature takes the finite value $1+E_{c} / E_{J}$. On the other hand, from (52) one finds in the limit of strong damping that the slope diverges as the temperature decreases and that the average number of excess Cooper pairs exhibits a jump of height one. This behavior should also be noticeable at very low but finite temperatures. From (50) we find for the slope of the step for weak damping $2 \hbar a<E_{J}$

$$
\chi=\frac{E_{c}}{E_{J}-2 \hbar a}
$$

which remains finite at zero temperature while for strong damping $2 \hbar a>E_{J}$ we get a slope of the form (62) which diverges as temperature goes to zero. A jump in the average number $\langle n\rangle$ of Cooper pairs on the island occurs in the limit of zero temperature only if the integrated impedance $\hbar a$ exceeds $E_{J} / 2$. In this case the jump of $\langle n\rangle$ is given by

$$
\Delta\langle n\rangle=\left[1-\left(\frac{E_{J}}{2 \hbar a}\right)^{2}\right]^{1 / 2}
$$

which continuously goes to zero as $\hbar a$ approaches $E_{J} / 2$ from above.

The dependence of the slope $\chi$ on the integrated impedance $a$ at low temperatures is shown in Fig. 5 . The dotted lines show the limiting cases $a=0$ and $a \rightarrow \infty$, respectively. The case of vanishing impedance yields a finite value for $\chi$ at zero temperature according to (43). For $E_{J} \rightarrow 0$ this always results in a jump as expected (see also Fig. 4). For infinite impedance $(a \rightarrow \infty) \chi$ diverges as the temperature decreases to zero according to (53). The curves in between, which have been obtained numerically from (50) with (21) and (16), show the behavior of $\chi$ at low temperatures for two intermediate values of the integrated impedance. The solid line for $\hbar a / E_{J}=0.2$ remains finite while the dashed line for $\hbar a / E_{J}=1$ diverges as temperature goes to zero according to our previous discussion.

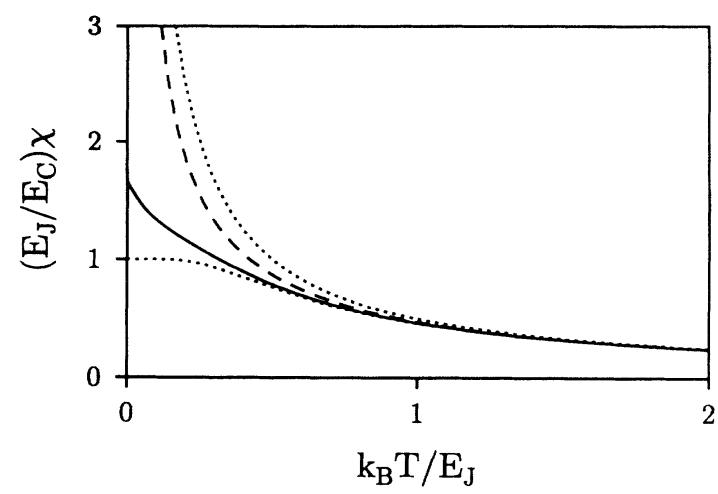

FIG. 5. Temperature dependence of the slope $\chi$ of the Coulomb staircase at $n_{x}=1 / 2$ for a low-frequency environment. The integrated impedance takes the values $\hbar a / E_{J}=0,0.2,1$, and $\infty$ from the lower to the upper curve. The lowest curve is obtained from (43) while the uppermost curve is obtained from (53). The two curves for finite impedance were calculated from (50) using (21) and (16).

\section{CONCLUSIONS}

We have determined the influence of the electromagnetic environment on the average number of Cooper pairs in a superconducting box. By mapping the Hamiltonian onto the spin-boson problem, we were able to calculate the partition function to all orders in the Josephson coupling energy for an electromagnetic environment described by an arbitrarily strong low-frequency impedance and a weak high-frequency impedance. It was found that the environment tends to suppress the charge fluctuations on the island. For a low-frequency environment the integral over the real part of the impedance turned out to be the relevant parameter. If the integrated impedance is larger than the Josephson coupling energy, the fluctuations due to Cooper pair tunneling are strongly suppressed. Even for finite Josephson coupling, a strong impedance at low frequencies may be sufficient to ensure a rather strict charge quantization at low temperatures.

\section{ACKNOWLEDGMENTS}

The authors would like to thank M. H. Devoret and D. Esteve for valuable discussions. This work was completed at the Centre d'Etudes de Saclay whose hospitality is gratefully acknowledged. Partial financial support was provided by the European Community under Contract No. SC1*-CT91-0631 and the Deutsche Forschungsgemeinschaft (DFG) through SFB237. One of the authors (G.L.I.) was supported by the DFG through the Heisenberg program.

\footnotetext{
* Present and permanent address: Institut für Physik, Universität Augsburg, Memminger Str. 6, D-86135 Augsburg, Germany.

${ }^{1}$ P. Lafarge, H. Pothier, E. R. Williams, D. Esteve, C.
}

Urbina, and M. H. Devoret, Z. Phys. B 85, 327 (1991).

${ }^{2}$ P. Lafarge, P. Joyez, D. Esteve, C. Urbina, and M. H. Devoret, Nature 365, 422 (1993).

${ }^{3}$ For a survey see Single Charge Tunneling, Coulomb Block- 
ade Phenomena in Nanostructures, edited by H. Grabert and M. H. Devoret, NATO ASI Series B, Vol. 294 (Plenum, New York, 1991).

${ }^{4}$ G.-L. Ingold and Yu. V. Nazarov, in Ref. 3.

${ }^{5}$ See, e.g., D. V. Averin and K. K. Likharev, in Ref. 3.

${ }^{6}$ For a study of these effects in normal conducting systems, see K. A. Matveev, Sov. Phys. JETP 72, 892 (1991) [Zh. Eksp. Teor. Fiz. 99, 1598 (1991)]; H. Grabert, Physica B, 194-196, 1011 (1994).

${ }^{7}$ D. Esteve, in Ref. 3.

${ }^{8}$ A. O. Caldeira and A. J. Leggett, Ann. Phys. (N.Y.) 149,
374 (1983).

${ }^{9}$ P. Lafarge, Ph.D. thesis, Université Paris 6, 1993.

${ }^{10}$ A. J. Leggett, S. Chakravarty, A. T. Dorsey, M. P. A. Fisher, A. Garg, and W. Zwerger, Rev. Mod. Phys. 59, 1 (1987).

${ }^{11}$ M. H. Devoret, D. Esteve, H. Grabert, G.-L. Ingold, H. Pothier, and C. Urbina, Phys. Rev. Lett. 64, 1824 (1990).

${ }^{12} \mathrm{H}$. Grabert and M. H. Devoret, in Ref. 3.

${ }^{13}$ R. Görlich and U. Weiss, Phys. Rev. B 38, 5245 (1988); R. Görlich, Ph.D. thesis, Universität Stuttgart, 1988. 